\title{
Vorwort zu Band 1883, Abth. I. u. II.
}

Während der Herausyabe des Bandes 1883 erkrankte der bisherige Redakteur der A bschnitte I. und II. Hr. Dr. Rosocisatius so schwer, dass er dauernd von der Herausgabe der Fortschritte zurticktreten musste. Um keine Stockung in dem Erscheinen, das an und fur sich durch versebicdene Umstände auch während der fruberen Redaktion verzögert war, eintreten zu lassen, ubernahm ich, auf Grund der für den zweiten Theil vorhandenen Vorarbeiten, die gesammte Redaktion auch flir Theil II. Es war erschienen Abth. I., die von Hrn. Dr. Rosochatius ganz allein redigirt ist, ebenso wie die ersten dreizehn Bogen der zweiten Abtheilung. Die dritte Abtheilung ist wie bisher unter derselben Leitung geblieben, Von 1884 an wird Hr. Dr. Budde die Redaktion der beiden ersten Abtheilungen übernebmen, und steht zu hoffen, dass das Ersebeinen der Bände ein schnelleres als bisher sein wird. Es liegt auf der Hand, dass es immerhin einige Schwierigkeiten haben wird, den Zusammenhaug zwischen den einzelnen Bänden herzustellen, da es sebr mulusam ist, festzustellen, wie weit die eine oder andere Arbeit schon fruber bertucksichtigt ist. Nur dadurch, dass der Unterzeichnete mit dem Geschäftsgange und der Einrichtung der Fortschritte seit langen Jahren vertraut war, ist es möglich gewesen, den Zusammenhang berzustellen und grössere Lücken zu vermeiden. Bei der Controlle leisteten auch die Beiblätter, herausgegeben von E. WiedEmann eine sehr schätzenswerthe Beihülfe und Erleichterung. - In der zweiten Abtheilung werden bei der Erklärung der Citate nur die Ergänzungen gegeben, und es sind hier auch die fur die dritte Abtheilung erforderlichen Erklärungen hinzugefugt. 
Was den Unfang der Fortschritte anbetrifft, so wächst die Litteratur so riesenhaft, dass es nicht möglich sein wird, die technischen Arbeiten mit pbysikaliscber Grundlage und ähnliche den benachbarten Gebieten (Physiologie, Astronomie, Mathematik, Geographie, Chemie u. s. w.) angehörende, vollständig zu berticksichtigen; es wird voraussichtlich eine Aenderung hierin nothwendig werden. Das Gebiet der Elektrotechnik allein wlirde schon einen Jahresbericht in Anspruch nehmen. Dabei wird die Zersplitterung der Journallitteratur immer grösser, da immer mebr gewisse Abzweigungen (Molekularphysik) ihre besondere Berlicksichtigung verlangen.

In Bezug auf diese und äbnlicbe Fragen, die sich in entsprechender Weise auf allen wissenschaftlichen Gebieten geltend machen, verweise icb auf eine kleine Scbrift, die in diesem Verlage erscheinen wird und gewissermaassen eine Erläuterung der Fortschritte darstellt, auf Grund der Erfahrungen, die nach beinahe 20 jähriger Redaktion gewonnen sind. Sie soll zugleich eine kurze bibliographische Zusammenstellung der Zeitschriften geben, welche flir die Fortschritte Berticksichtigung erfahren haben und also Arbeiten physikalischer Natur enthielten.

Ftr diesen Jabrgang habe ich, da ich die Redaktion von II. nur interimistisch ubernommen habe und der Band schon im Druck war, geglaubt nicht kürzen zu durfen; daher ist die Elektrotechnik nach den vorliegenden Referaten und einer Litteraturzusammenstellung mit bertteksichtigt. Auch habe ich den Gedanken des Hrn. Dr. Rosochatius, den geschichtlicben Theil binzuzunehmen auf Grund der Vorarbeiten zur Ausfthrung gebracht, freilich konnte bier nur der Litteraturnachweis gegeben werden.

Bei den Referaten ist der Gedanke fest gehalten, dass dieselben möglichst die Originalarbeit in der Form geben, dass die erstere für die Leser nicht unumgäuglich nothwendig ist; andererseits sollte der Vollständigkeit durch möglichst ausgedebnte und ubersichtliche Litteraturangaben Rechnung getragen werden.

Prof. Dr. B. Schwalbe. 